\title{
Paediatric dental A\&E service during the COVID-19 pandemic in the Greater London area
}

\author{
N. Patel ${ }^{1} \cdot$ A. Viswanathan ${ }^{1} \cdot$ J. Lee ${ }^{1} \cdot$ S. Barrow ${ }^{1} \cdot$ A. Cant $^{1} \cdot$ R. Sanghvi ${ }^{1} \cdot$ M. Deseta $^{1} \cdot$ J. Layton $^{1} \cdot$ N. Bhujel ${ }^{1}$. \\ E. C. Sheehy ${ }^{1}$
}

Received: 19 September 2020 / Accepted: 18 November 2020 / Published online: 3 January 2021

(c) European Academy of Paediatric Dentistry 2021

\begin{abstract}
Purpose The UK government introduced a nationwide lockdown on the 23rd March 2020 to prevent the spread of COVID19. All elective hospital and dental practice assessments and procedures were mandated to stop. Key hospital dental workers were required to work, and Guy's and St Thomas' NHS Foundation Trust became a designated Urgent Dental Care Centre (UDC) for the greater London area. The paediatric dental emergency walk-in service was suspended and replaced with a telephone triage system and evaluation of digital images sent by parents/carers when needed. The aim of this paper is to describe the emergency service provided by staff in the department of Paediatric Dentistry at St Thomas' Hospital during the first lockdown.

Methods A prospective service evaluation of the modified paediatric dental emergency service was carried out between 25th March and 29th May 2020.

Results Four-hundred and sixty-four patients accessed the paediatric dental emergency service via telephone during the service evaluation period. Of these, $192(41 \%)$ had dental pain, 121 (26\%) had pain and swelling of dental origin, and 89 $(19 \%)$ had trauma.

Conclusions Remote telephone consultations and digital photographs were useful to screen emergency paediatric dental patients, but lack of face-to-face consultations with radiographic assessment and access to general anaesthetic services were major limiting factors.
\end{abstract}

Keywords COVID-19 · Paediatric dental accident and emergency

\section{Introduction}

An outbreak of pneumonia cases in Wuhan, Hubei Province, China was reported by the Chinese Centre for Disease Control and Prevention in January 2020. The causative organism was identified as a coronavirus, SARS-CoV-2, and the disease was named COVID-19 by The World Health Organisation (WHO) (Zheng 2020). The aetiology of COVID-19 is zoonotic in origin and it can be transferred from person to person via droplet spread through coughing and sneezing. It can also spread through contact with mucous membranes such as oral, nasal and eye mucosa and bodily fluids such as saliva and contaminated surfaces (Peng et al. 2020).

N. Patel

Nikita.patel1@gstt.nhs.uk

1 Guy's and St Thomas' Hospitals NHS Trust, St Thomas' Hospital, Westminster Bridge Road, London SE1 7EH, UK
COVID-19 has had a profound impact on the dental profession. Everyday dental treatment involves the generation of splatter and aerosols of bodily fluids including blood and saliva, which increases the likelihood of transmission of viruses such as SARS-CoV-2 (Ilyas et al. 2020). The use of dental equipment such as high-speed handpieces, 3-in-1 air-water syringes, and suction aspirators initiates an aerosol generating procedure (AGP) which form part of everyday dental care. Thus, dentists are at an increased risk of viral exposure through inhalation of virus particles during AGPs (Sabino-Silva et al. 2020).

On 23rd March 2020, the United Kingdom government announced strict "lockdown" measures to prevent the spread of the infection; and on 25th March 2020, the Chief Dental Officer of England issued the requirement to stop all elective assessments and non-urgent dental care (GOV.UK 2020; Hurley and Neligan 2020). Practices were asked to commence a remote urgent care service 
utilising telephone triage before arranging face-to-face appointments. Urgent dental care (UDC) hubs were gradually set up which included 36 in the South-East of England region and over 30 in the East of England. These hubs were designed to meet the urgent dental needs of the local population whilst maintaining social distancing guidance, providing appropriate personal protective equipment (PPE), and preventing patients travelling long distances during the lockdown period (NHS East of England 2020; NHS South East 2020).

The COVID-19 pandemic and subsequent closure of primary care dental practices had a profound impact on the provision of care in the hospital settings. Routine dental care for patients was deferred, and urgent dental care was prioritised.

The aim of this paper is to describe the emergency service provided by staff in the department of Paediatric Dentistry at St Thomas' Hospital during the first lockdown.

\section{Methods}

This prospective study was registered as a service evaluation with the Trust Clinical Governance Unit in accordance with the local governance protocol (Ref Number 10775/10788). The service evaluation involved all patients who accessed our paediatric dental emergency service via telephone or in-person from 25th March 2020 to 29th May 2020. The paediatric dental accident and emergency walk-in service was suspended during the initial lockdown period and replaced with a telephone triage system, whereby all patients were required to contact the department by telephone. Once registered, the details of each patient were then passed on to a dentist to be triaged. The triage process involved the use of a dental emergency proforma (Fig. 1), medical history, and relevant COVID-19 questionnaires. These were developed using guidance from the Royal College of Surgeons of England and Scottish Dental Clinical Effectiveness Programme (RCSEng 2020; SDCEP 2020).
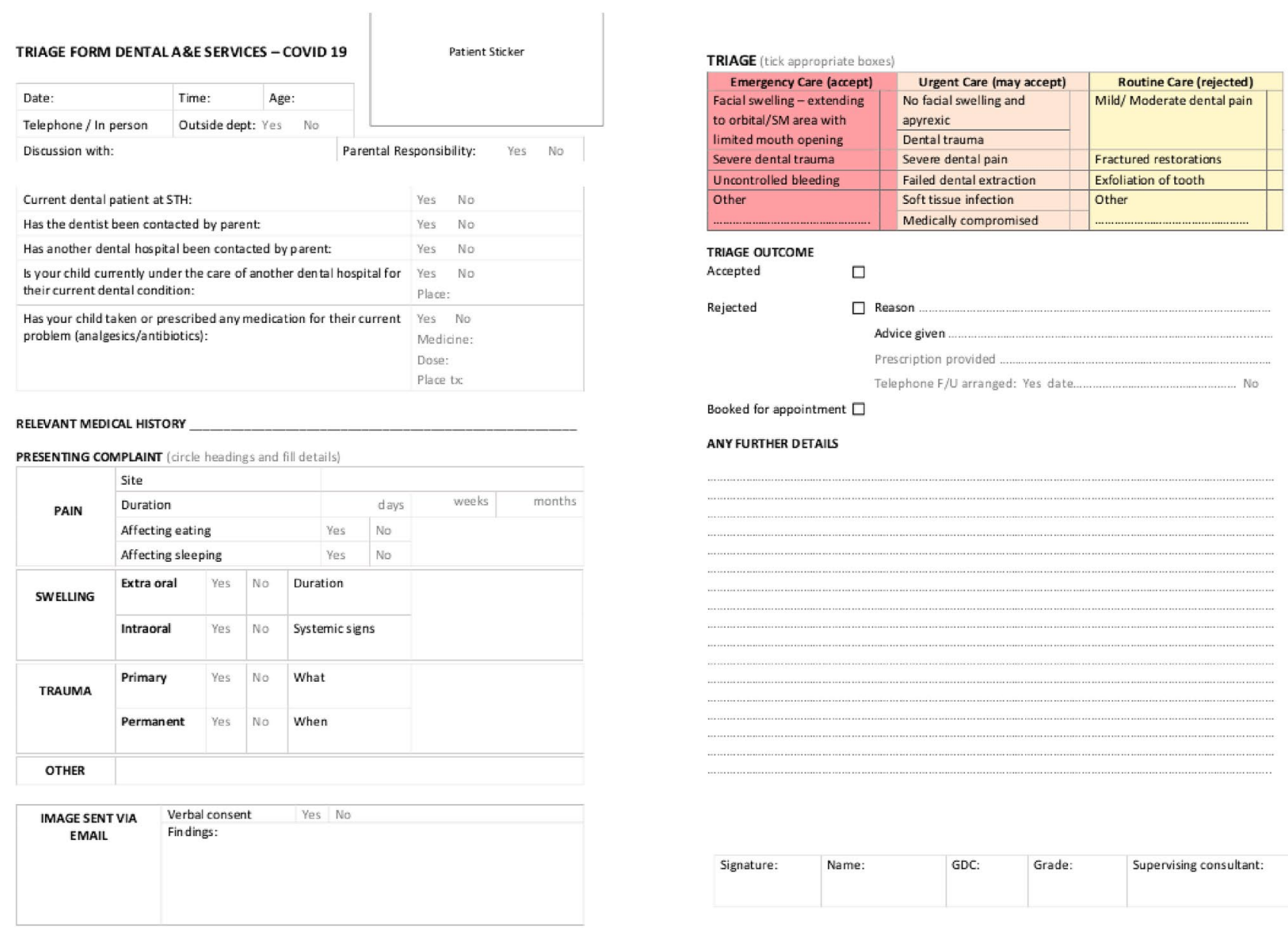

Fig. 1 Dental accident and emergency proforma used during COVID-19 pandemic 
Parents were asked to send digital photographs of their child's dental concern (e.g., extra-oral or intra-oral swellings, teeth causing pain, or trauma) to a secure mailbox when needed to aid the telephone triage assessment.

Following this, cases would be designated to one of the following triage outcomes based on their dental need and their medical history: emergency, urgent, or routine. All cases classified as emergencies were accepted for face-toface appointments or immediately referred to the patient's local hospital if more appropriate, especially if admission of the patient in an Oral and Maxillofacial Surgery (OMFS) unit was deemed appropriate. Urgent cases had an appointment arranged in the department or were referred to the patient's nearest UDC or advised to phone their local general dental practitioner (GDP). Routine cases were given reassurance, advice about pain management, and parents were also informed on the protocol for seeking advice if the symptoms were to worsen or change. Remote prescriptions, the majority of which were antibiotics, were arranged for patients via their general medical practitioner (GMP) or GDP, for collection at their local pharmacy.

Each case that was triaged by the department was logged using a secure data collection tool in Microsoft Excel, and the data recorded were: patient age, referral source, reason for accessing the emergency service, and triage outcome.

\section{Results}

Four-hundred and sixty-four patients accessed the paediatric dental emergency service via telephone during the service evaluation period. The details of the patient data included in this service evaluation are shown in Table 1 . The majority who contacted the department were parents of the paediatric patients $(n=453,97 \%)$, whilst the other $3 \%$ constituted other family members $(n=5), 4$ GDPs, and two consultant paediatricians from The Evelina London Children's Hospital (ELCH). Most telephone triages were carried out by the junior dental team $(n=420,91 \%)$, which is composed of dental core trainees, specialty dentists, and speciality registrars. The remaining telephone consultations $(n=44,9 \%)$ were completed by consultants or specialists in paediatric dentistry. Care plans for patients were made jointly by the junior dental member and a consultant or a specialist in paediatric dentistry.

The most common reason for accessing the dental emergency service was dental pain only $(n=192,41 \%)$, followed by dental pain with intra-oral and/or extra-oral swellings $(n=121,26 \%)$. Trauma to either primary or secondary teeth was the chief complaint of 89 patients (19\%). Of these patients, 37 sustained trauma to their permanent teeth, 45 to their primary teeth, and 7 patients had an injury to the soft tissues only. Other dental issues such as broken removable
Table 1 Patient data related to Paediatric Dental A\&E calls during the COVID-19 lockdown

\begin{tabular}{ll}
\hline Variable & $\begin{array}{l}\text { Number } \\
\text { of patients } \\
(\%)\end{array}$ \\
\hline Caller & \\
Parent & $453(97)$ \\
GDP & $4(1)$ \\
ELCH & $2(1)$ \\
Other & $5(1)$ \\
Responder & \\
Junior & $420(91)$ \\
Consultant or specialist & $44(9)$ \\
Reason for call & \\
Advice & $12(3)$ \\
Pain & $192(41)$ \\
Pain and swelling & $121(26)$ \\
Trauma & $89(19)$ \\
Query waiting list times & $28(6)$ \\
Other & $22(5)$ \\
COVID-19 risk assessment results & \\
High risk & $6(1)$ \\
Low risk & $160(35)$ \\
Not known & $298(64)$ \\
\hline
\end{tabular}

appliances, oral ulceration, lost restorations or mobile primary teeth accounted for 22 patients (5\%), and small proportion of patients, $(n=28,6 \%)$ were from parents querying appointments and waiting times for general anaesthesia. The remaining calls $(n=12,3 \%)$ were to seek advice about the patients' referral pathways, especially if patients were experiencing dental pain during the lockdown, or to enquire about the longevity of temporary restorations.

Two-hundred and eighty-two (61\%) patients were managed remotely over the telephone by the provision of advice for pain management; home use of temporary restoration kits for minor trauma to primary and permanent teeth; as well as oral health instructions. Approximately a fifth of the patients $(n=96,21 \%)$ were advised to seek care from local GDP's, GMP's or UDCs. These patients either required remote antibiotic prescriptions $(n=41)$ to be collected from their GDP/GMP, to seek care from local GDP once they resumed normal practice $(n=9)$ or to attend their local UDCs for assessment and treatment $(n=46)$. After completing the telephone triage, 86 patients $(18 \%)$ were accepted for face-to-face appointments and dental care in the department.

A variety of treatments were carried out on the patients that attended, as shown in Table 2. The majority of these were carried out by consultants $(n=82,95 \%)$, three patients (4\%) patients were treated by a specialist, and one patient $(1 \%)$ by a specialty registrar in paediatric dentistry. We were able to utilise the GA priority list at ECH for 11 of patients 
Table 2 Treatment provided during the lockdown period

\begin{tabular}{lc}
\hline Treatment & $\begin{array}{c}\text { Number } \\
\text { of cases }\end{array}$ \\
\hline Advice only & 10 \\
Fluoride varnish & 1 \\
Prescription & 8 \\
Primary tooth extraction & 26 \\
Permanent tooth extraction & 7 \\
Trauma—dressing/Cvek/extirpation & 21 \\
Caries—dressing & 7 \\
Admitted/GA planned & 11 \\
Resin bonded bridge/appliance repair & 2 \\
\hline
\end{tabular}

and one of these patients had a positive COVID-19 swab result. The parents of five children declined to come to the hospital for face-to-face appointments, and therefore, they were given an outcome of "advice only".

During the different stages of lockdown we managed an influx of patients from boroughs outside of our catchment area and outside of London, as depicted in Fig. 2. From 14th April, the data collection form was modified to include how the patients heard about our emergency service. The mode of referral was recorded for 223 patients. Fifty-six percent $(n=125)$ were instructed by NHS Dental 111 to contact us for advice, GDP's directly referred
24 patients (11\%), and 3 patients (1\%) were referred by community dental practices. Twenty-five percent $(n=57)$ of parents said that they had found our contact details online or from previous correspondences, and other parents $(n=14,7 \%)$ were recommended to contact us by their GMP's, friends or other family members. Only $19 \%$ $(n=86)$ of patients who contacted us were registered patients of St Thomas' paediatric dental department.

There were six patients $(1 \%)$ in total who answered 'yes' to one or more questions in our COVID-19 questionnaire. Four of these patients were given advice remotely, including to contact NHS 111 for COVID-19 specific advice, and one parent was advised to seek care in their local community practice. One patient was advised to come for assessment the following day as the family would have completed their 14 days of isolation. As the majority of patients $(n=298,64 \%)$ could be managed over the phone with advice, they were not asked to complete the COVID-19 screening questionnaire.

An upward trend was noticed in the number of calls over the lockdown period as shown in Fig. 3. The peak number of calls was 100 calls a week and 30 calls a day during the eighth week into the lockdown period. We moved towards a more streamlined way of accepting patients for the remaining two weeks of the lockdown and noticed a fall in the number of patients requiring consultation.
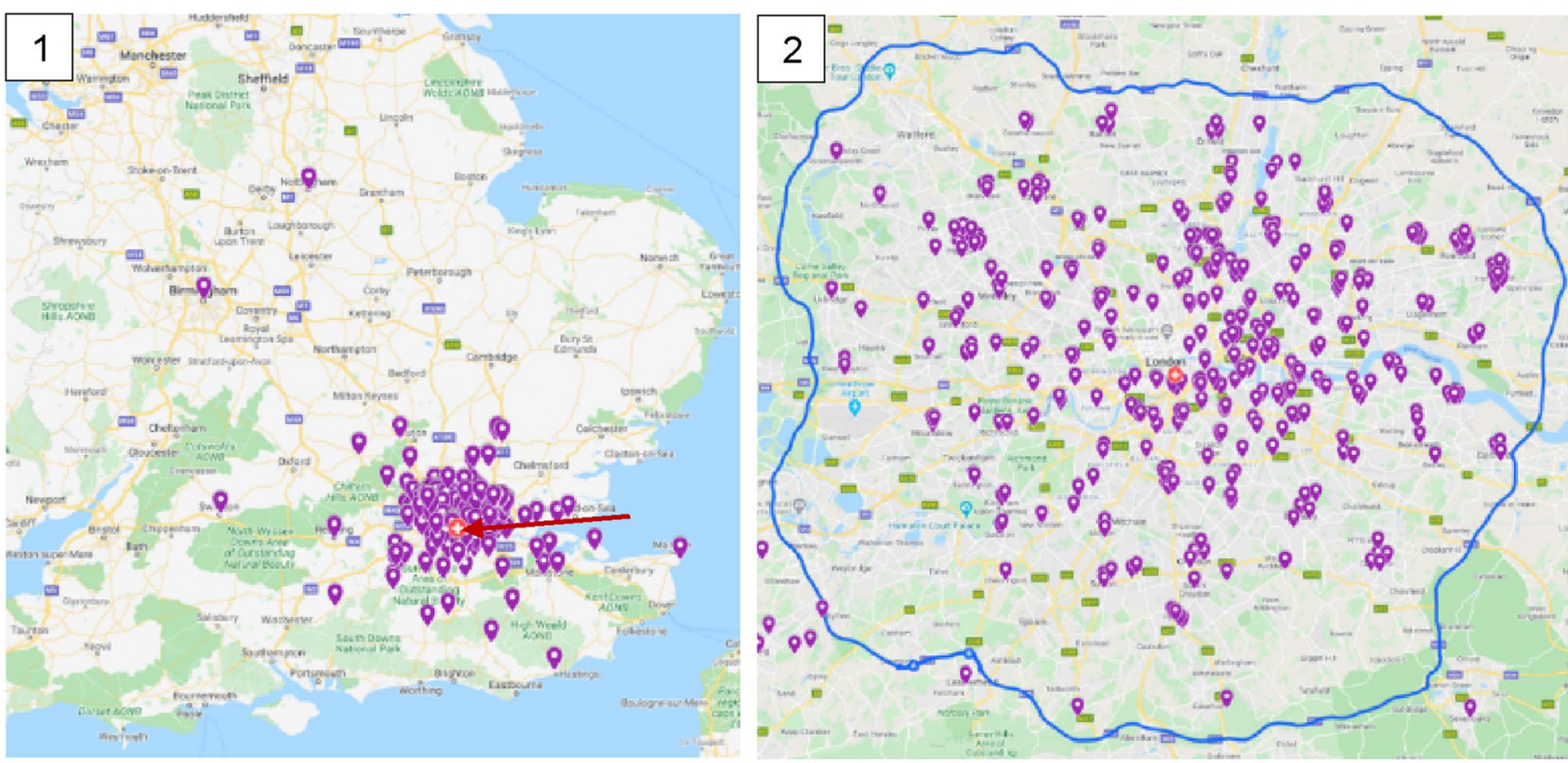

Fig. 2 The distribution of patients who contacted the department across the country are shown in map 1. A detailed map of the patients within the boundaries of Greater London and the location of our department (red cross) can be seen in map 2 
Fig. 3 The number of calls received over the 10 -week lockdown period

\section{Number of Calls During Covid 19 Lockdown}

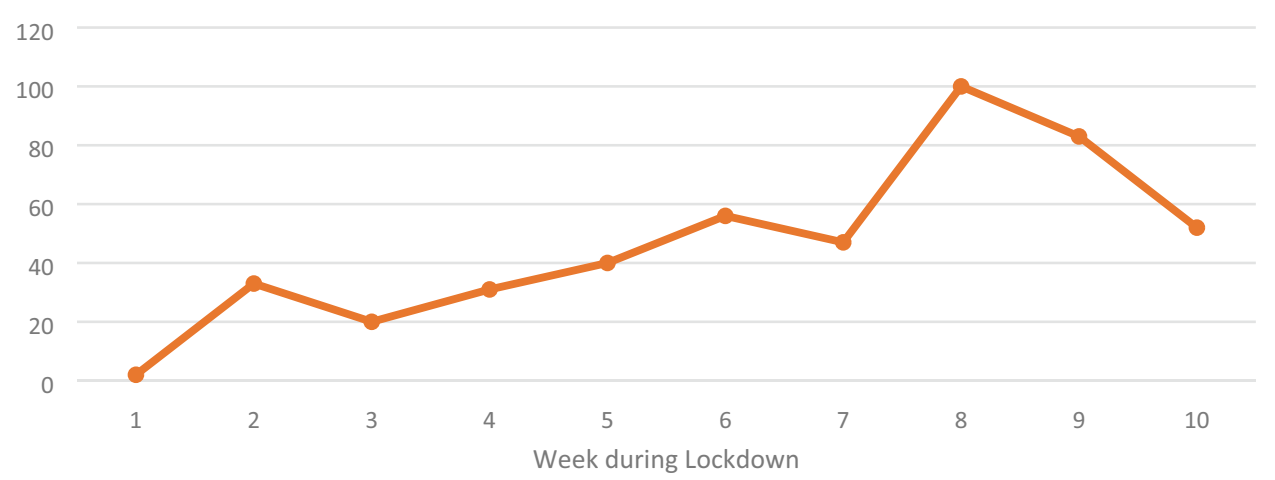

\section{Discussion}

This service evaluation highlights the impact of the COVID19 pandemic on our paediatric dental service and how the department adapted to the changes. The service we provided reflected the guidance set out in March 2020 by the Royal College of Surgeons of England, Scottish Dental Clinical Effectiveness Programme, and Royal College of Paediatric and Child Health (RCSEng 2020; SDCEP 2020; RCPCH 2020). Our aim was to treat patients appropriately whilst minimising face-to-face contact to reduce the spread of COVID-19.

In the early stages of the lockdown, we noted a decrease in the number of paediatric patients seeking urgent dental care within our department. This trend had also been reported by accident and emergency (A\&E) departments across the country, which saw the number of visits drop by $49 \%$ during lockdown (Thornton 2020). This was most likely due to the public's initial reluctance to leave their homes as they were fearful of contracting COVID-19. However, as the lockdown progressed, the number of telephone calls received for dental emergencies steadily increased. This year, in the period of March to June, the number of patients accessing our paediatric dental emergency service was 2.4 times higher than in the same period last year. This can be explained by our service running as a UDC, and therefore, we spoke to more patients outside our catchment area. Although UDCs were set up in and around London, patients were still struggling to access their local service. Rapid changes were needed to cope with the significant increase in demand for the service, whilst ensuring the department was compliant with government and public health guidelines.

Throughout the pandemic, we found no notable difference in the types of conditions that presented at the paediatric dental emergency clinic compared to pre-COVID-19. Toothache was the most common reason for patients seeking urgent care, which once again highlights the significant burden of dental caries, not just on the quality of life and development of the child, but also on the health system (Public Health England 2017). Dental trauma is another common reason for paediatric patients to seek dental care and we triaged many of these patients. With each case, we followed guidance set out by the British Society of Paediatric Dentistry on trauma during the COVID-19 pandemic (Day et al. 2020a, b).

Management of acute dental problems in young children can be particularly challenging, because they tend to cooperate poorly in the dental chair for the procedures that would remove the source of pain and infection (e.g., extraction or extirpation). Pre-COVID-19, we could offer the option of general anaesthetic for some patients. However, suspension of all elective general anaesthetic services was advised to allow for staff and resources to be redirected to prioritised areas during the pandemic (NHS England 2020). Hence, most of the non-emergency toothache cases had to be managed solely with the three A's principle-advice, analgesia, and antimicrobials where appropriate (SDCEP 2020). Understandably, this was not always received well by some parents, who wanted a more definitive treatment to alleviate their child's pain. Clear and empathetic communication was vital to address their concerns, particularly since the mode of communication was by telephone and most parents were distressed and anxious about seeking dental care during the pandemic.

If urgent care was deemed necessary, prior to entry into the department, all patients were screened for symptoms of COVID-19 using our screening questionnaire. Paediatric patients with COVID-19 often had mild or no symptoms, and therefore, all patients entering the department were treated as asymptomatic carriers (RCSEng 2020). It was recommended that the temperature of each patient and accompanying person should be checked prior to faceto-face assessment in the dental chair; and for paediatric patients, only one parent or guardian could attend the dental clinic to minimise the risk of exposure. In addition, AGPs were avoided where possible. Each clinical situation was 
risk-assessed and PPE was provided according to government advice. For non-AGPs, this included eye protection, a disposable fluid-resistant (Type llR) surgical mask, a disposable apron, and gloves. For AGPs, disposable fluid-repellent gown, gloves, eye/face protection, and an FFP3/N95 respirator mask were worn by those carrying out or assisting in the procedure (NHS England and OCDO England 2020).

We relied on carrying out treatment in clinic for emergency and urgent patients, because access to GA was limited. Patients who were deemed as requiring emergency/ urgent care under GA following guidance from the Royal College of Surgeons of England were admitted and were treated as asymptomatic carriers of the infection. The hospital's structured protocol ensured safe and successful delivery of care to these patients. Initially, there was no routine testing at ELCH and this progressed to testing for patients, and as the situation changed, patients had a swab test for COVID-19 on admittance to the hospital.

A less common reason for patients accessing the dental emergency service during the lockdown period was oral ulceration. A 9-year-old boy who presented with oral ulceration on the lower lip had a complex medical history including severe dystonia and epilepsy. This patient was later diagnosed with a paediatric inflammatory multisystem syndrome, temporarily associated with SARS-COV-2 (PIMSTS) (ECDC 2020). We later learnt that all of the patients that were admitted to ELCH with PIMS-TS had oral symptoms. Although COVID-19 usually causes a mild infection in children and many remain asymptomatic, dental practitioners should remain vigilant and consider COVID-19 as a potential causative factor in paediatric patients presenting with oral and mucosal symptoms which are common in viral infections.

Co-operation with other health professionals across the NHS was crucial for successful management of patients remotely during the pandemic. For example, following triage if the patient required antibiotics, to avoid patients having to travel great distances to St Thomas' Hospital, antibiotic prescriptions were arranged in liaison with the patient's GDP for collection at their local pharmacy. For those not registered with a dentist, their GMP was contacted instead. However, some challenges encountered included the increased time required to make these arrangements and e-mailing prescriptions, as well as the reluctance of some GMPs to prescribe antibiotics for dental reasons, because they were concerned that it was outside their scope of practice. To ensure that these patients were followed up appropriately, all patients who were prescribed antibiotics had telephone reviews 3-5 days later with either our department or by their local GDP.

Communication with other health professionals was also key when we received calls of medically compromised patients requiring treatment and paediatric patients with large facial swellings requiring intravenous antibiotics, incision and drainage, and extractions. We liaised closely with the patient's nearest OMFS on-call service to ensure they were treated promptly without unnecessary excess travel. A list of surrounding OMFS on-call services and Community Dental Services with appropriate contact numbers was compiled for the department.

One of the main challenges we faced with the adapted service was operating a triage service remotely and digitally. As face-to-face consultations had to be kept to a minimum, photographs were used to supplement the history taken by telephone to make a diagnosis and subsequently determine the most appropriate management. Telemedicine had previously been described in the literature as a useful tool for diagnosing and managing patients in disasters and public health emergencies, and COVID-19 has certainly accelerated the adoption of this new technology in dentistry (Lurie and Carr 2018). However, actual implementation of telemedicine did have several obstacles.

Firstly, data protection and patient confidentiality had to be secure throughout, especially when using photographs for the triage process. To ensure this, parents were requested to send photographs to secure NHS mailboxes only. Secondly, language barriers were even more challenging when telephone was the only mode of communication. To reduce the risk of miscommunication, Language Line services were used via a three-way call system. However, this service was not always readily available, especially for urgent cases. Therefore, there were occasions where communication had to be primarily with another member of the household who spoke English; and in these cases, photographs were especially useful to aid the diagnosis. Thirdly, the telephone consults were mostly with the parents or guardians of the children, and not with the child themselves. This may arguably affect the reliability of the history of presenting complaints. As well as this, we are trained to be vigilant regarding any safeguarding concerns with children that present to our service. Operating a digital service may reduce our awareness of these patients.

Despite the limitations, we found that telephone consultations with photographs were effective and beneficial to the emergency triage service during the lockdown period. Ultimately, it allowed patients and their parents or guardians to consult with health care professionals from the safety of their own homes.

\section{Conclusions}

The COVID-19 global pandemic has caused major disruption to dental services and those provided by the Paediatric Dentistry Department at St Thomas' Hospital. During this time, the department ensured patients received the necessary 
advice and care whilst rapidly adapting to guidance. There were prompt alterations to routine practice, standard operating procedures, and use of PPE. In these times, the swift dissemination of new guidance and modification to services has relied on the flexibility of services and staff. In future, better guidance and improved communication from the governing bodies would be vital to improve access to care and patient safety and to prevent services becoming overwhelmed.

The use of teledentistry is likely to be more widely adopted in dentistry, but it should not be considered a substitute for a thorough face-to-face consultation, radiographic assessment, and treatment planning of our patients. However, its usefulness was clearly beneficial during this time.

Acknowledgements The authors would like to acknowledge the dental team in the paediatric dental department at St Thomas' Hospital, for their work and support of the emergency dental service in the department. All authors approve the manuscript and no funding was required for this service evaluation.

Author contributions All authors contributed to data collection and write up of the paper.

Funding Not applicable.

Data availability Not applicable.

Code availability Not applicable.

\section{Compliance with ethical standards}

Conflict of interest The authors declare that they have no conflict of interest.

\section{References}

Day P, Djemal S, Albadri S. Primary Dentition Acute Management of Traumatic Injuries and Follow-up Care during the COVID19 Pandemic. British Society of Paediatric Dentistry and Dental Trauma UK; 2020a. Available from: https://www.dentaltrauma.co. uk/File.ashx?id=15336. [cited 2020 Jul 31]

Day P, Djemal S, Albadri S. Permanent Dentition Acute Management of Traumatic Injuries and Follow-up Care during the COVID-19 Pandemic [Internet]. British Society of Paediatric Dentistry and Dental Trauma UK; 2020b [cited 2020 Jul 31]. Available from: https://www.dentaltrauma.co.uk/File.ashx?id=15336

ECDC. Paediatric inflammatory multisystem syndrome and SARS$\mathrm{CoV}-2$ infection in children. European Centre for Disease Prevention and Control; 2020. Available from: https://www.ecdc.europ a.eu/sites/default/files/documents/covid-19-risk-assessment-paedi atric-inflammatory-multisystem-syndrome-15-May-2020.pdf. [cited 2020 Jul 31]

GOV.UK. Coronavirus (COVID-19). 2020. Available from: https:// www.gov.uk/coronavirus. [cited 2020 May 11]
Hurley S, Neligan M. Issue 3, Preparedness letter for primary dental care. 2020. Available from: https://www.england.nhs.uk/coron avirus/wp-content/uploads/sites/52/2020/03/issue-3-preparedne ss-letter-for-primary-dental-care-25-march-2020.pdf. [cited 2020 Jun 4]

Ilyas N, Agel M, Mitchell J, Sood S. COVID-19 pandemic: the first wave - an audit and guidance for paediatric dentistry. Br Dent J. 2020;228(12):927-31.

Lurie N, Carr BG. The Role of Telehealth in the Medical Response to Disasters. JAMA Intern Med. 2018 01;178(6):745-6.

NHS East of England. Urgent dental care for patients in the East of England. 2020. Available from: https://www.england.nhs.uk/ east-of-england/2020/04/30/urgent-dental-care-for-patients-inthe-east-of-england/. [cited 2020 Jul 31]

NHS England. Next Steps on NHS Response to COVID-19. NHS England and NHS Improvement; 2020. Available from: https://www. england.nhs.uk/coronavirus/wp-content/uploads/sites/52/2020/03/ urgent-next-steps-on-nhs-response-to-covid-19-letter-simon-steve ns.pdf. [cited 2020 Jul 31]

NHS England, OCDO England. Dental standard operating procedure: Transition to recovery [Internet]. NHS England and NHS Improvement; 2020 [cited $2020 \mathrm{Jul}$ 31]. Available from: https:// www.england.nhs.uk/coronavirus/publication/dental-standardoperating-procedure-transition-to-recovery/

NHS South East. Urgent Dental Care in the South East. 2020. Available from: https://www.england.nhs.uk/south-east/2020/04/17/urgen t-dental-care-in-the-south-east/. [cited 2020 Jul 31]

Peng X, Xu X, Li Y, Cheng L, Zhou X, Ren B. Transmission routes of 2019-nCoV and controls in dental practice. Int J Oral Sci. 2020;12(1):9.

Public Health England. Health matters: child dental health. 2017. Available from: https:/www.gov.uk/government/publications/ health-matters-child-dental-health/health-matters-child-denta 1-health. [cited 2020 Jul 31]

RCPCH. COVID-19- guidance for paediatric services. Royal College of Paediatrics and Child Health; 2020. Available from: https:// www.rcpch.ac.uk/sites/default/files/generated-pdf/document/ COVID-19---guidance-for-paediatric-services.pdf. [cited 2020 May 11]

RCSEng. Recommendations for Paediatric Dentistry during the recovery phase of the COVID-19 pandemic. The Royal College of Surgeons of England; 2020. Available from: https://www.rcseng.ac. uk/dental-faculties/fds/coronavirus/. [cited 2020 Jul 1]

Sabino-Silva R, Jardim ACG, Siqueira WL. Coronavirus COVID-19 impacts to dentistry and potential salivary diagnosis. Clin Oral Investig. 2020;24(4):1619-21.

SDCEP. Management of acute dental problems during COVID-19 Pandemic. Scottish dental clinical effectiveness programme; 2020. Available from: https://www.sdcep.org.uk/wp-content/ uploads/2020/03/SDCEP-MADP-COVID-19-guide-300320.pdf. [cited 2020 Jun 4]

Thornton J. Covid-19: A\&E visits in England fall by $25 \%$ in week after lockdown. BMJ. 2020;6(369):m1401.

Zheng J. SARS-CoV-2: an emerging coronavirus that causes a global threat. Int J Biol Sci. 2020;16(10):1678-85.

Publisher's Note Springer Nature remains neutral with regard to jurisdictional claims in published maps and institutional affiliations. 